VIRIS SVMME REVERENDIS

IISDEMQVE ILLVSTRISSIMIS ATQVE OMNIBVS

HVMANITATIS DOTIBVS CVMVLATIS

\title{
CAPITVLI ECCLESIAE EPISCOPALIS VERONENSIS PRAESIDI ET SACERDOTIBVS
}

\author{
HEGIA SCIENTIARYM ACADEMIA BORVSSICA \\ S. P. D.
}

En Vobis Vestrum, Viri Summi, Gajum, quem ıostrorum curne et fidei commisistis, reddimus: et talem quidem reddimus, quale $m$ sperabamus Vobis gralissimum fore. Nam cum Dei providentia et majorum merito domini et dispensatores facti sitis supellectilis litterariae cum copia conspicuae tum praestantia paene incomparabilis, Vos tanto thesauro non incubatis, res ad erudiendum genus humanum utilissimas non premitis neque eas orbi litterato quam primum impertiendi laudem cuiquam, modo digno et ad id negotium apto, invidetis: sed illud polius e re Vestra, illam Vestram maximam laudem putatis, si Vestrae tantae opes ho- 
die polius quam cras ila publicenlur, ul ad Dei immortalis gloriam, ad verae scienliae amplificalionem, ad bonorum ingeniorum alimentum, quam plurimus inde fructus redundet.

Quare cum Augustissimi Regis nostri ad Summum Pontificem oralor, collega nosler, B. G. Nieburus, vir ad excolenda el adjuvanda oplima sludia nalus, urbem Vestram ante hos qualtuor annos Iransirct celeberrimamque illam bibliothecam invisere cuperel; Vos tanquam Vestro eam aperuistis et permisistis ul, quae vellel, diligentius ille inspicerel et transcriberel. Ita factum est ut codicis illius preliosissimi, cujus particulam jam vir ille doclissimus, quem suum olim fuisse Vestra Verona merilo sibi gralulalur, Scipio Muffeius, veluti augurans quanti thesauri primilias orbi litterato offerret, publici juris fecerat, cjus inquam codicis ceterae quoque partes abscondilae hucusque et alius operis characteribus obrulae, studio et sagacitate viri optimi detegerenlur. Neque hic substitit Vester erga bonas artes favor; neque Vos thesaurum hunc, quod alii fecissent, nunc aut multo magis anxie secludebalis, aut vana Vos patriamque celeriori quam solidiori honore maclandi cupidilale abrepti, minus exercilatis manibus adornandum tradebalis: sed cum audissetis esse apud nos viros aliquos, qui hoc polissimum jurium antiquitalis studium, 
quod saeculo proximo per universam Europam marcesceliat, strenue instanrarent; non dubilastis, quae Vestra est animi magniludo, viris extraneis, quos nos Vobis commendaveramus, copiam facere scrinia Vestra adeundi, codicem in orbe terrarum sui generis unicum manibus tractandi, el cousque illi insudandi, donec omnia quac cemporis injuria non plane evanuerint, in charlas suas inde conferrent. gua in re officia alque indefessum studium benevolenliamque Vestri Bibliohecarii, Viri reverendissimi el illustrissimi, Bartholomaci Comilis Guarienti, qui nos omnes co munere sibi devinxil, gratissima memoria recolere solent. Postremo humanissime eos dimisistis animoque aequissimo el benignissimo concessistis, ut apographum illud atro gemmisque acquiparandum domum asportarent uberiori hic otio perpoliendum ct, quod jam peractum videlis, cum orbe erudito communicandum.

Ilaque Vobis, Viri Summi, id acceplum debelur, quod Gaji opus illud, ex quo jam olim, vigente Romano imperio, a doctoribus elementa Iuris Romani tradebantur, postquam per tot saecula e rerum natura deletum creditum est, nunc denuo eidem gravissimac scientiac tradendae in nostris etiam scholis inservit. Vestro merilo debetur inventum, quod in maximis inde ab instauratione litterarum est nume- 
randum. Vestrum est quod nova lux his litteris affulsit, quodque velut nova aetas jurisprudentiae Romanae nunc surgit. Quas igitur tota Vobis respublica litteraria gratias debet, eas patimini ut nos potissimum Vobis agamus, quos in meriti illius consortium quadamtenus recipere dignati estis, cum viros a nobis ad Vos missos tanta et humanitate et liberalitate ad honorificentissimum illud munus admitteretis.

Cujus meriti quam vellemus ut re quoque par Vobis referre contingere!! Interim quae nobis relicta est, publicam hanc sensuum nostrorum testificationem, et, doni instar, illud ipsum quod Vos nobis donaslis, benigne ut accipialis rogamus enixe.

Dabamus, Berolini mense Novembri Anni MDCCCXX.

\author{
PAVLVS ERMAN \\ FRIDERICVS DANIEL ERNESTVS \\ SCHLEIERMACHER \\ IOANNES GEORGIVS TRALLES \\ PHILIPPVS BVTTMANN
}

